\title{
Nephron sparing endoscopic treatment for primary carcinoma of the renal calyx: A case report and literature review
}

\author{
QI WANG ${ }^{1}$, TONG-WEN OU ${ }^{1}$, JIA-WEI XU ${ }^{1}$, JIN LI $^{1}$, ALI BORAZJANI ${ }^{2}$, \\ CHUN-SONG JIA ${ }^{1}$, XU WANG ${ }^{1}$ and HAO YAN ${ }^{1}$ \\ ${ }^{1}$ Department of Urology, Xuanwu Hospital, Capital Medical University, Beijing 100053, P.R. China; \\ ${ }^{2}$ Department of Biomedical Engineering, Lerner Research Institute, Cleveland Clinic, Cleveland, OH 44195, USA
}

Received October 29, 2015; Accepted April 15, 2016

DOI: $10.3892 / \mathrm{mco} .2016 .867$

\begin{abstract}
Primary carcinoma of the renal calyx is extremely rare. The present study reported nephron sparing endoscopic treatment for primary carcinoma of the renal calyx. An 81-year-old female presented with a 1-year history of intermittent painless gross hematuria. Computed tomography and X-ray of the urinary tract were unable to definitively identify any lesion. Flexible ureteroscopic examination revealed a tumor with epicenter in the lower calyx of the right kidney, with additional involvement around the calyx. Biopsies were obtained and pathology revealed low-grade urothelial carcinoma. Considering additional co-morbidities, the patient elected to undergo endoscopic management with thulium laser. The present report described the feasibility of flexible ureteroscopic thulium laser resection for the treatment of renal calyx carcinoma.
\end{abstract}

\section{Introduction}

Renal calyx carcinoma ( $\mathrm{RCXC}$ ) is a novel concept, which is defined by Williams et al (1) as carcinoma developing primarily from the renal calyx. Recent advances in endourologic techniques have resulted in increased popularity of endoscopic management of upper tract urothelial carcinoma (UC) (2-4). To the best of our knowledge, no previous reports have presented treatment of RCXC patients with nephron-sparing endoscopic surgery. The present study reported the use of a flexible ureteroscopic strategy for the resection of RCXC with a thulium laser in an 81-year-old female presenting with a 1-year history of intermittent painless gross hematuria.

Correspondence to: Dr Hao Yan, Department of Urology, Xuanwu Hospital, Capital Medical University, 45 Changchun Street, Beijing 100053, P.R. China

E-mail: yanhurology@qq.com

Key words: calyx, carcinoma, renal pelvis, thulium laser, ureteroscopic surgery

\section{Case report}

An 81-year-old female presented at hospital with intermittent painless gross hematuria and was treated with oral antibiotics for 1 week; the gross hematuria subsequently resolved. At $\sim 1$-year later, the patient again presented at the same hospital with painless gross hematuria. Four days later, the patient came to the Emergency Department, Xuanwu Hospital (Beijing, China) complaining of acute pain in the right waist and back. Urinary tract ultrasound and contrast-enhanced urinary tract computed tomography (CT) revealed hydronephrosis of the right upper urinary tract. No stone was observed on X-ray or CT, however, CT revealed a small suspicious mass in the lower calyx of the right kidney (Fig. 1). Furthermore, the patient underwent cystoscopy, and no evidence of bladder carcinoma or stone was found. However, the urine ejected from the right ureteral orifice was noted to have a reddish tint compared with the left ureteral orifice. Renal function tests revealed a blood creatinine of $114 \mu \mathrm{mol} / 1$, right renal glomerular filtration rate (GFR) of $32.6 \mathrm{ml} / \mathrm{min}$ and left renal GFR of $21.7 \mathrm{ml} / \mathrm{min}$. No evidence of metastasis were observed on the bone scan and chest X-ray. At 6 days later, the patient underwent a flexible ureteroscopic examination performed under general anesthesia, and a $9 \mathrm{~mm}$ tumor with an epicenter in the lower renal calyx of the right kidney was detected, along with additional small lesions around the calyx (Fig. 2). Biopsies were obtained and sent for pathological examination, which revealed low-grade UC (Fig. 3). Following discussions with the patient and her family, particularly with regards to other co-morbidities, including renal insufficiency, coronary heart disease, hypertension, diabetes and advanced-age, it was decided to proceed with endoscopic management using thulium laser.

At 4 days after the initial ureteroscopy, the patient underwent endoscopic resection of the RCXC under general anesthesia with flexible ureteroscopy in the lithotomy position. The laser energy setting was set to $9 \mathrm{w}$, and saline was used for irrigation. Following entering the pelviureteric junction, the ureteroscope was carefully guided to the mass in the lower calyx of the right kidney using pre-operative CT imaging. Subsequently, the thulium laser was utilized to firstly ablate the margins of the renal sinus fat. Next, the entire tumor was resected to a depth of $4 \mathrm{~mm}$ using laser fulguration. The 


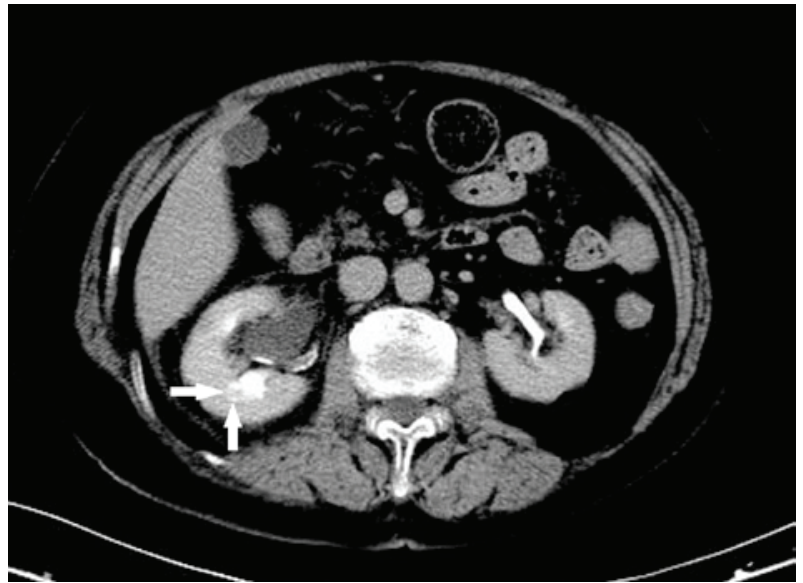

Figure 1. Computed tomography shows hydronephrosis of the right renal pelvis and a small suspicious mass (white arrow) in the lower calyx of the right kidney.

A

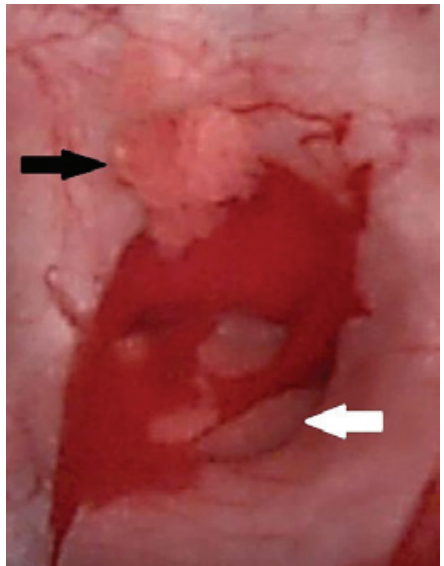

B

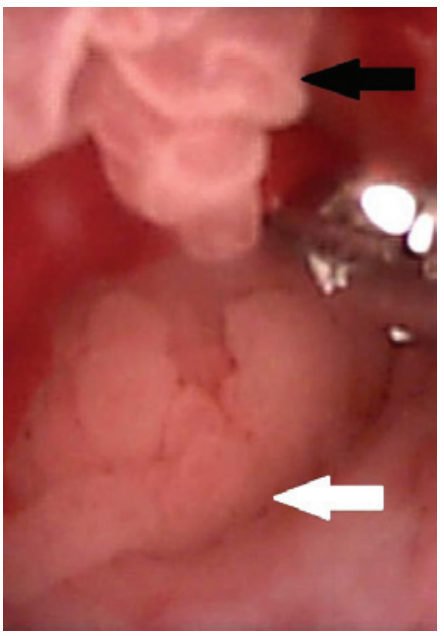

Figure 2. (A) Distant and (B) close view of flexible ureteroscopic examination shows the main tumor (white arrow) with epicenter in the lower renal calyx of the right kidney and additional small lesions (black arrow) around the calyx.

wound was irrigated with $100 \mathrm{ml}$ of $0.1 \mathrm{mg} / \mathrm{ml}$ epirubicin in saline, followed by irrigation with $500 \mathrm{ml}$ distilled water. The other calyxes were examined using flexible ureteroscopy to confirm that no tissue was left behind. Finally, a $4.7-\mathrm{Fr}$ double J ureteral stent was placed in the right urinary tract. The total surgery duration was $66 \mathrm{~min}$. The double J ureteral stent

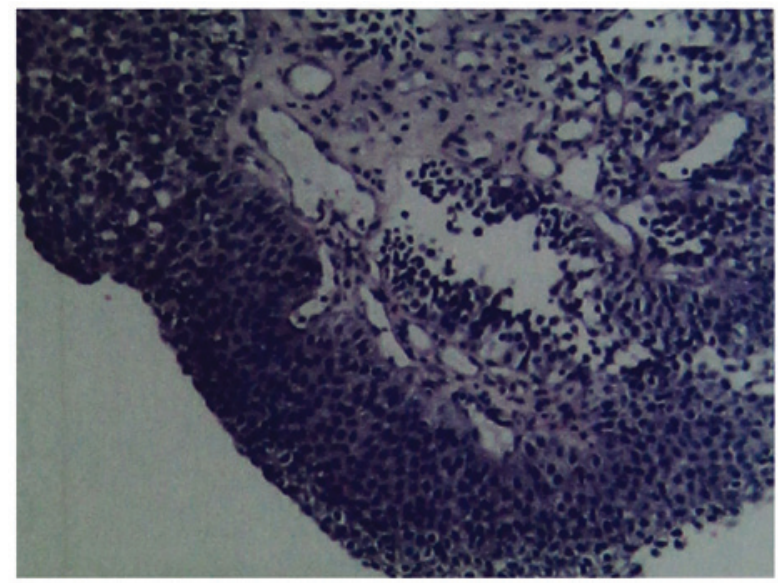

Figure 3. Pathology shows low-grade urothelial carcinoma following staining with hematoxylin and eosin;. magnification, x200.

was removed 1-month later and no recurrence was detected at the 6-month follow-up.

\section{Discussion}

The collecting duct and the urothelium of the upper urinary tract system share similar embryological origins (5). However, the histopathological features of UC of the renal pelvis and collecting duct carcinoma (CDC) are quite different. Williams et al (1) were the first to present the concept of RCXC in 2013. RCXC can be either CDC or UC of the renal pelvis, and compared with traditionally defined upper tract urothelial carcinomas, RCXC has a locally aggressive behavior, which may limit its spread into the urothelium of the urinary tract (1). However, traditional imaging techniques and rigid ureteroscopic examinations typically fail to detect such lesions in the early stage (6).

Although traditional radical nephroureterectomy with bladder cuff excisions remains the gold standard surgical treatment for upper tract urothelial carcinoma (UTUC) (4), this procedure may result in morbidity and loss of nephron units (3). As endourologic techniques have developed further, nephron-sparing procedures (NSPs) have become the first-line management strategy for selected patients with low grade UTUC and are feasible to complete resection or fulguration. Management with NSPs consists of ureteroscopic or percutaneous approaches (4), and the technical feasibility and safety of the NSPs have been shown in several previous studies $(3,7)$. Potential candidates for NSPs include those with low risk UTUC who have a normal contralateral kidney or those patients with serious comorbidities (i.e. patients with a solitary kidney or renal insufficiency) (4). In the present study, the patient's bilateral renal GFR was $54.3 \mathrm{ml} / \mathrm{min}$, which is indicative of renal insufficiency. In addition, the diameter of the tumor was $\sim 9 \mathrm{~mm}$ and the lesion was located in a renal calyx with limited involvement of the surrounding renal pelvis urothelium, and the histopathological findings revealed low-grade urothelial carcinoma. All of these features were consistent with UTUC that is low-risk. Following communication with the patient and her family, a conservative endoscopic surgical approach followed by long-term endoscopic surveillance was selected. 
Previously, several adjuvant topical agents, including Bacillus Calmette-Guérin (BCG), mitomycin C, epirubicin and thiotepa, have been used for UTUC perfusion treatment $(4,8)$. Benefits of the antegrade perfusion with BCG in patients with UTUC following ablation of early stage tumors has also been demonstrated (8). Nonetheless, certain controversy remains regarding the safety and efficacy of retrograde instillations, which may cause pyelovenous influx and ureteric obstruction during perfusion (4). However, in the present case, the entire operation was performed using flexible ureteroscopy, and it was not believed to be worthwhile to make additional incisions to enable antegrade perfusion. Therefore, the present study selected retrograde perfusion with epirubicin immediately following the resections. Following perfusion with epirubicin, distilled water was used to wash the renal pelvis and flexible ureteroscopy was used to check that no residual tissue remained in other renal calyces.

In conclusion, flexible ureteroscopy with thulium laser is a novel nephron sparing endoscopic treatment for primary carcinoma of renal calyx. This procedure is feasible for select patients who are willing to undergo long-term endoscopic surveillance as follow-up.

\section{References}

1. Williams PA and Mai KT: Primary carcinoma of renal calyx. Pathol Res Pract 209: 654-661, 2013.
2. Rouprêt M, Traxer O, Tligui M, Conort P, Chartier-Kastler E, Richard $\mathrm{F}$ and Cussenot $\mathrm{O}$ : Upper urinary tract transitional cell carcinoma: Recurrence rate after percutaneous endoscopic resection. Eur Urol 51: 709-713; discussion 714, 2013.

3. Yakoubi R, Colin P, Seisen T, Léon P, Nison L, Bozzini G, Shariat SF and Rouprêt M: Radical nephroureterectomy versus endoscopic procedures for the treatment of localised upper tract urothelial carcinoma: A meta-analysis and a systematic review of current evidence from comparative studies. Eur J Surg Oncol 40: 1629-1634, 2014

4. Rouprêt M, Babjuk M, Compérat E, Zigeuner R, Sylvester RJ, Burger M, Cowan NC, Böhle A, Van Rhijn BW, Kaasinen E, et al: European Association of Urology Guidelines on Upper Urinary Tract Urothelial Cell Carcinoma: 2015 Update. Eur Urol 68: 868-879, 2015.

5. Bohnenpoll T and Kispert A: Ureter growth and differentiation. Semin Cell Dev Biol 36: 21-30, 2014.

6. Yamany T, van Batavia J, Ahn J, Shapiro E and Gupta M: Ureterorenoscopy for upper tract urothelial carcinoma: How often are we missing lesions? Urology 85: 311-315, 2015.

7. Hoffman A, Yossepowitch O, Erlich Y, Holland R and Lifshitz D: Oncologic results of nephron sparing endoscopic approach for upper tract low grade transitional cell carcinoma in comparison to nephroureterectomy-a case control study. BMC Urol 14: 97, 2014.

8. Giannarini G, Kessler TM, Birkhäuser FD, Thalmann GN and Studer UE: Antegrade perfusion with bacillus Calmette-Guerin in patients with non-muscle-invasive urothelial carcinoma of the upper urinary tract: Who may benefit? Eur Urol 60: 955-960, 2011. 